\title{
Onset of Synaptogenesis in the Plexiform Layers of the Chick Retina: A Transmission Electron Microscopic Study
}

\author{
ULRICH DRENHAUS, * TILMAN VOIGT, AND GÜNTER RAGER \\ Department of Medicine, Division of Anatomy, University of Fribourg, Fribourg, Switzerland

\begin{abstract}
KEYWORDS gapjunction; chemical synapses; development; ultrastructure
\end{abstract}
\begin{abstract}
The presently acknowledged onset of synaptogenesis in the chick retina from embryonic day 12 (E12) onward stands in contrast with the appearance of spontaneous electrical activity, of presynaptic proteins, or of neurotransmitters during early formation of the inner (E6-E8) and outer (E9) plexiform layers. Therefore, we investigated the chick retina from E6 to E12 at which age first synapses appear by transmission electron microscopy (TEM). The study provides evidence that synaptogenesis in the chick retina begins shortly after the plexiform layers have started to emerge. The first synapses are electrical synapses, which appear on E7, one day after the future inner plexiform layer emerged, and towards the end of E8 in the nascent outer plexiform layer. Conventional chemical synapses appear in both plexiform layers on E8, in the inner plexiform layer (stage 34) only a few hours earlier than in the outer plexiform layer (stage 35). The first synapses are formed close to the apex of the optic fissure and their frequency increases rapidly with age. The onset, the topography, and the developmental course of synaptogenesis correlate with the chronotopic course of maturation of retinal neurons and the age when spontaneous electrical activity occurs in the retina.
\end{abstract}

\section{INTRODUCTION}

The rationale of this study is a phenomenon reported for all vertebrates investigated so far: The discrepancy between the first appearance of electrical activity and of synaptic contacts in the developing neuronal retina. A paradigm is the intensively studied embryonic chick retina with its particularly broad and well-demarcated layers. There, spontaneous electrical activity arises on embryonic day 7 or 8 (E8), and from E9 on, synchronized bursting activity, associated with calcium waves, is a robust event in most embryos (Catsicas et al., 1998; Sernagor et al., 2000; Wong et al., 1998). The activity is thought to be driven and modulated by synaptic release of neurotransmitters as in other vertebrates (Catsicas and Mobbs, 2001; Feller et al., 1996; Sernagor and Grzywacz, 1996; Zhou, 1998). However, morphological evidence for the presence of chemical synapses at these ages is lacking. In the inner plexiform layer (IPL), which starts to form between E6 and E8 (Drenhaus et al., 2003; Prada et al., 1999; Spira et al., 1987), transmission electron microscopic (TEM) studies ascertained conventional synapses from E12/13, and dyad synapses from E14 onward (Hughes and LaVelle, 1974; Sheffield and Fischman, 1970). In the outer plexiform layer (OPL), which becomes visible on E9 (D'Alessandri et al., 1995; McLaughlin, 1976), the beginning of synaptogenesis is unknown. Triad synapses, characteristic for photoreceptor cells, occur from E15/16 onward (Hughes and LaVelle, 1974; McLaughlin, 1976).

The absence of chemical synapses directed the attention to electrical synapses (maculae communicantes, nexus, or gap junctions). In the chick retina, gap-junctional blockers suppress spontaneous activity suggesting that the activity is triggered and propagates by mechanisms that involve neurotransmitters and gap junctions including the possibility of neurotransmitter coupling through gap junctions (Catsicas et al., 1998; Vaney et al., 1998; Wong et al., 1998). However, TEM studies did not detect gap junctions in the embryonic plexiform layers of the chick retina during the period under question. Fujisawa et al. (1976b) described gap junctions at early embryonic stages. But from E7.5 onward, their number decreased with the depth of the neuronal retina and they became predominantly restricted to cell surfaces close to the outer limiting membrane, and they disappeared eventually on E9. Connexin immunohistochemistry suggests, however, that gap junctions are present in the inner part of the retina at the time when the plexiform layers arise, increase in number until about E14 and decrease then with the onset of synaptogenesis (Becker et al., 2002).

During the last decade, essential information about the development of the retina accumulated. Today, it is well established that the development of both plexiform layers, their differentiation into sublayers, and the maturation of retinal neurons start in general in the central part of the retina close to the optic fissure and proceeds from there into the periphery (Drenhaus et al., 2003; Prada et al., 1991, 1999). Presynaptic proteins are present concurrent with the forming IPL and they spread following the spatial course of the maturating retina (Bergmann et al., 1999, 2000; Hering and Kröger, 1996). Glutamine synthetase activity suggests the onset of synaptogenesis in the OPL on E9 (Prada et al., 1998). Consequently, one may assume that synaptogenesis

*Correspondence to: Dr. Ulrich Drenhaus; Department of Medicine, Division of Anatomy, Rte A. Gockel 1, CH-1700 FRIBOURG, Switzerland. E-mail: ulrich.drenhaus@unifr.ch 
starts much earlier than presently acknowledged and that this process begins in the central retina. As the onset of synaptogenesis is a key event in the development of the neuronal retina and a pivotal reference for different processes such as formation of neuronal circuits or structural patterning, we reinvestigated in E6-E12 chick embryos by TEM at which age first synaptic contacts are formed in both plexiform layers. Thereby, we focused on the region close to the optic fissure.

\section{MATERIALS AND METHODS}

Twenty-one E6-E12 day old White Leghorn chick embryos of Hamburger-Hamilton (1951) stages HH28 $(n=1), 30$ (1), 31 (2), 34 (1), 35 (6), 36 (7), 37 (1), and HH38 (2) were investigated. Before fixation, all embryos received a lethal dose of the anesthetic Vetanarcol ${ }^{\circledR}$ (Veterinaria AG, Zürich, Switzerland) by intraperitoneal injection of $3.5 \mathrm{mg}$ Pentobarbitalum natricum $/ \mathrm{g}$ body weight. The fixative was perfused (2.5\% glutaraldehyde, $1 \%$ PFA in $0.1 \mathrm{M}$ phosphate buffer adjusted to $\mathrm{pH} 7.4$ ). The dissected eyes were flat mounted and the central region comprising the optic fissure surrounded by about $2 \mathrm{~mm}$ of retinal tissue was cut out. These specimens were postfixed with $2 \% \mathrm{OsO}_{4}$, dehydrated, and embedded as described earlier (Drenhaus et al., 2000). The polymerized resin blocks were cut with the Reichart Ultracut S ultra microtome (Leica, Wien, Austria) using diamond knives. The sections were orientated longitudinally to the retinal layers and perpendicular to the longitudinal axis of the optic fissure and comprised all layers. Semithin sections, stained with toluidin blue, served for proper orientation of the section plane. Series of ultrathin sections (thickness: $70 \mathrm{~nm}$ ) were prepared comprising 15-62 sections in the stages HH28-HH35 and 31-68 sections in the stages HH36-HH38. They were mounted on mash or on single-whole copper grids covered with a supporting film (1\% formvar dissolved in dioxane) and stained with uranyl acetate and lead citrate. In some sections, the staining was preceded by treatment with $0.25 \%$ potassium permanganate.

For examination, the TEM Zeiss EM 10C (Carl Zeiss AG, Zürich, Switzerland) was used which was equipped with the electro-magnetic stage control device "Otikom" (Zeiss) that was connected to a computer and allowed to record the position of the specimen stage within a preestablished Cartesian system of $\mathrm{x}$ and $\mathrm{y}$ coordinates to register the perimeter coordinates of the plexiform layers and to record the exact position of micrographs or synaptic contacts, respectively. The micrographs were scanned using the Imacon Flextight Precision 949 scanner and FlexColor software 3.9.3 (Imacon A/S, Copenhagen, Denmark) and fed into the computer to identify and to measure the size of the contacts using Adobe Photoshop CS.

\section{RESULTS HH28-HH30 (E6)}

First sprouting ganglion cell dendrites were visible on HH30, which did, however, not yet form a continuous layer (not shown).

\section{HH31 (E7)}

The IPL appeared as a small continuous stripe. Cell processes, which might belong to amacrine cells, joined the dendrites of ganglion cells. Frequently dark stained opposed membranes of various length (range: 0.09$0.22 \mu \mathrm{m})$ occurred between these processes. In the smaller junctions sometimes tiny annular structures were visible adjacent to these membranes to which also amorphous material was associated at the cytoplasmic side (Fig. 1A). The opposed membranes were separated by a small cleft filled with electron-dense material that appeared partially condensed to minute structures crossing the intercellular gap. The larger junctions were less frequent. They differed from the smaller junctions in the thickness of the opposed membranes, which were accompanied by a dark rim of opaque material on the cytoplasmic side nearly along their whole extension. In some junctions, microfilaments infiltrated the opaque material and occasionally a dense core vesicle located in the neighborhood (Fig. 1B).

\section{HH34 (E8-E8.5)}

The above described two types of junctions in the IPL were more frequent and some of them were elongated, particularly those which resembled the type shown in Figure 1A. The appearance of the other type (Fig. 1B) was as before. In addition, rare contacts occurred with small vesicle-like structures associated with the cytoplasmic densities (Fig. 1C). Furthermore, clusters of vesicle-like structures were observed without synapse-like specializations in the neighborhood or on adjacent sections (not shown, but see Fig. 2D). Such clusters occurred also at later stages.

\section{HH35 (E8.5-E10)}

The IPL had become much broader and the packing of processes was tighter. Junctions similar to those found on E7 (Fig. 1A) were increased in size and appeared band-like straightened or slightly curved. The distance between the opposing membranes was reduced, was of constant width throughout, and was filled with an opaque substance (Fig. 1D). The electron dense material on the cytoplasmic side adjacent to the opposed membranes was not interspersed with filamentous material and these junctions were still devoid of synaptic vesicles. The appearance and the frequency of the other type of junctions found on E7 (Fig. 1B) were as before. Since this remained until E12 we will not refer to this type in the following text. Contacts that displayed synapse-like specializations contained a few more vesicle-like structures at the end of E8 (Fig. 1E) and their number increased during E9 (Fig. 1F). At the end of E9, some contacts incorporated dense core vesicles (Fig. 1G). At this age, the frequency of contacts was increased and they appeared to be clustered, particularly in the outer and inner part of the IPL.

A nascent OPL was present towards the end of E8. At that age, junctions were found (Figs. 2A and 2B) resembling those seen also early in the developing IPL. The gaps between the opposed membranes were filled with electron dense material of varying density and occasionally a subendoplasmalemmal undercoating by an opaque line was visible (Fig. 2A). A few hours later, some of the junctions were considerably elongated (Fig. 2D). Simultaneous with the first junctions, a very small number of contacts occurred that showed synapse-like structures including a few vesicle-like structures associated to one of the opposing membranes (Fig. 2C). On 

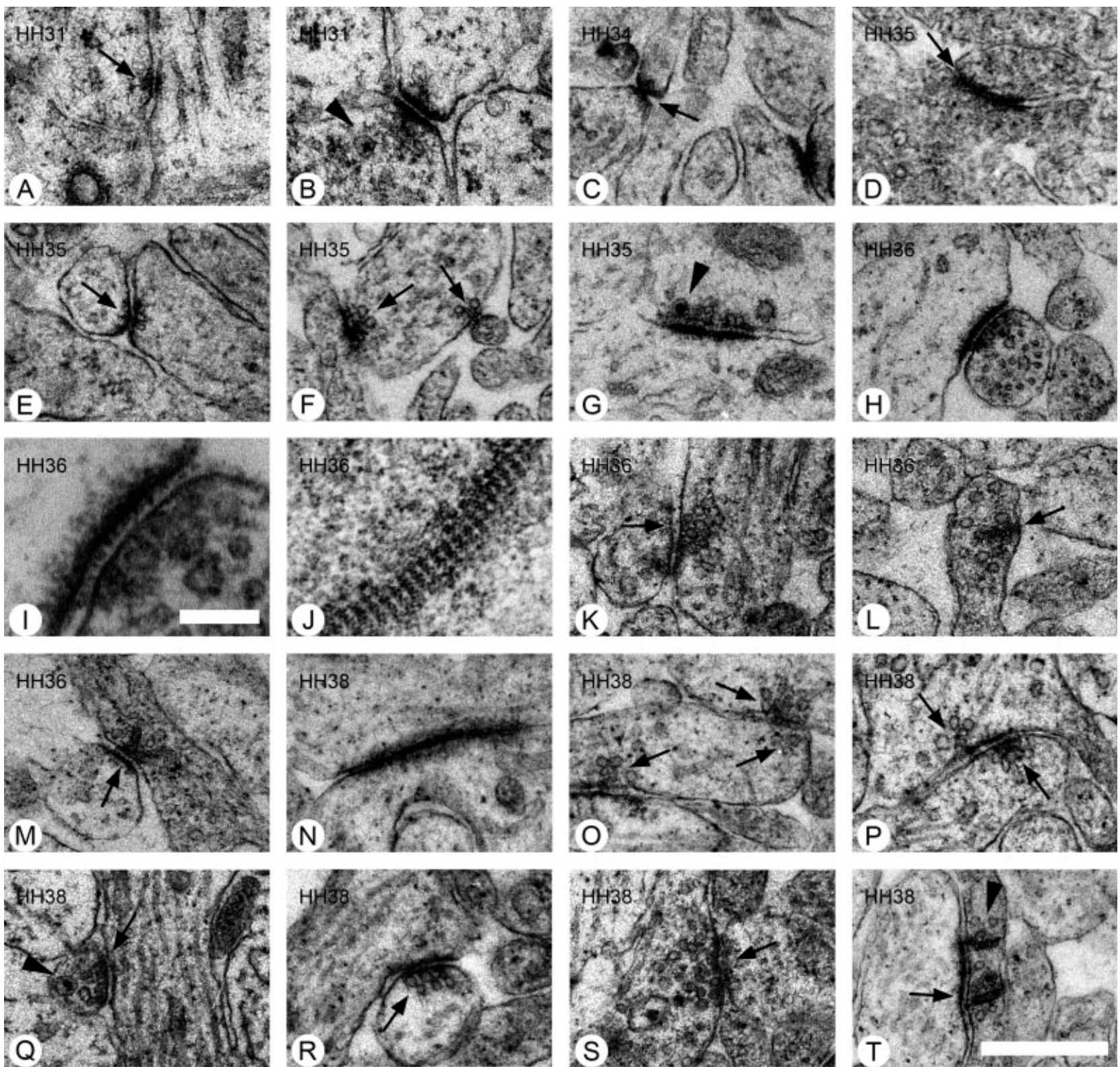

Fig. 1. Electron micrographs of the chick IPL in the region close to the apex of the optic fissure from HH31 (E7) until HH38 (E12). A, B: At E7.3, first intercellular contacts occurred. One type was small (A, arrow) and the opposed membranes were less prominent compared to the larger second type (B), having an opaque rim adjacent to the opposed membranes to which some fibrous material was associated at the cytoplasmatic side and a dense core vesicle (arrowhead). C: At early E8, contacts appeared which showed prominent membrane thickenings (arrow) accompanied by a few vesicle-like structures. D-F: At the transition from E8-E9, junctions resembling the type shown in A were elongated and the distance between the opposing membranes was reduced (D, arrow). The contacts with synapse-like specializations showed an increasing number of vesicle-like structures (E, F, arrows); in some contacts dense core vesicles were present (G, arrowhead). H-J: At the end of E10, junctions resembling those observed earlier were found to be enlarged. When cut oblique, structures became visible within the gap between the opposed membranes $(\mathrm{H}$; enlarged detail in I). Occasionally a regular lattice pattern of

intermembrane particles was visible $(\mathrm{J})$ which consisted of small pores arranged in parallel, partially curved rows. K: Contacts with synapse-like specializations showed an increased number of vesiclelike structures. L, M: On E10, contacts with synaptic ribbons appeared (arrows) which were already accompanied by vesicles. Some of the contacts displayed already dark stained opposed membranes (M). N-T: On E12, the junctions were even more increased in length $(\mathrm{N})$ and they were always devoid of vesicles. Contacts with synapselike specialization (O-S, arrows) appeared more mature and varied in their appearance. Some processes showed two contacts in close neighborhood $(\mathrm{O})$, and occasionally reciprocal synapses were found $(\mathrm{P})$ Other variations refer to the size and shape of the vesicle-like structures, the presence of dense core vesicles $(\mathrm{Q}$, arrowhead), and the appearance of densities at the contact zone. Contacts with synaptic ribbons had increased ribbons and in most cases dark stained membranes at the opposite surface ( $\mathrm{T}$, arrow). Rarely a conventional synapse occurred in the neighborhood (T, arrowhead). Scale bar in I $=0.1 \mu \mathrm{m}$, for all other images in $\mathrm{T}=0.5 \mu \mathrm{m}$. 

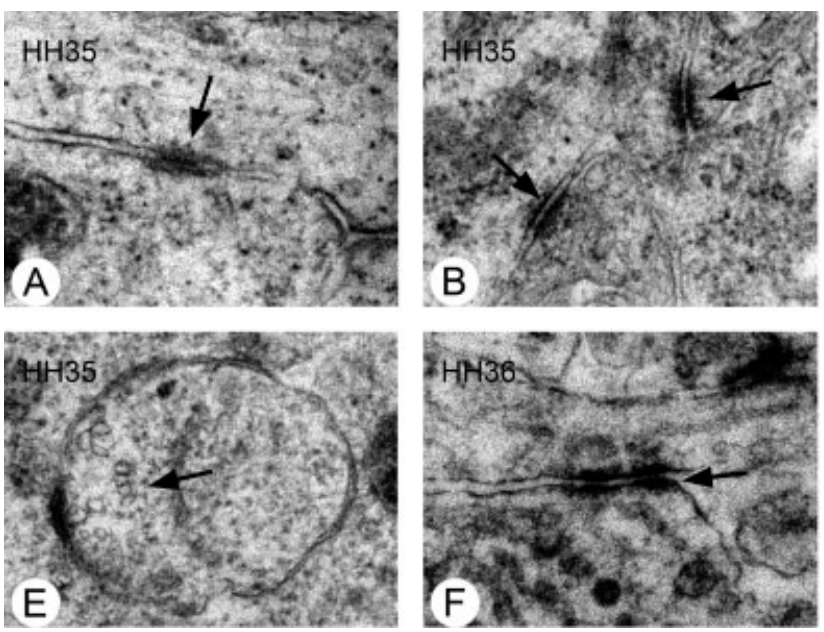

Fig. 2. Micrographs of the chick OPL close to the apex of the optic fissure at HH35 (E8.5, E9) and HH36 (E10). A-C: At the transition from E8 to E9 junctions occurred (A, B; arrows) which resembled those found in the IPL. Note the subplasmalemmal undercoating by electron dense material in A (arrow). Simultaneous contacts were present which displayed synapse-like specializations (C, arrow). D-E: In the middle of E9, junctions (D, arrows) were elongated and the gap was reduced compared to earlier stages. Frequently, accumulations of vesicle-like structures were seen (D, arrowhead) which were not

E9.5, the number of such contacts was increased and some of them displayed more vesicle-like structures (Fig. 2E). Frequently, accumulated vesicle-like structures were seen in the absence of synapse-like specializations (Fig. 2D), as already reported for the IPL at HH34. They, too, occurred also at later stages as in the IPL.

\section{HH36, HH37 (E10, E11)}

Many of the junctions described earlier (Figs. 1A and 1D) were increased in size. When cut oblique, intermembrane particles of rather regular distance were visible in the gap between the opposed membranes (Figs. $1 \mathrm{H}$ and 1I). In suitable section planes, a regular lattice pattern of dark stained clusters appeared consisting of small annular structures or pores arranged in parallel, partially curved rows (Fig. 1J). The maximum extension measured up to $2.9 \mu \mathrm{m}$. The distance between the clusters amounted to $\sim 50-55 \mathrm{~nm}$ and the center-to-center distance between the pores within the clusters varied between $5-13 \mathrm{~nm}$. The diameter of the pores was determined at about $2 \mathrm{~nm}$. The frequency of contacts with synapse-like specializations was increased and many displayed larger numbers of vesicle-like structures (Fig. 1K). On E10, a different type of contact occurred in the IPL characterized by a ribbon-shaped density accompanied by vesicle-like structures on either side of the ribbon (Figs. 1L and $1 \mathrm{M})$. The length of the ribbon-shaped density varied between 90-100 nm. In most cases, the adjacent membrane was not particularly pronounced, but in very few cases this membrane appeared darkened and the opposed membrane of the adjacent cell process was thickened (Fig. 1M).

In the developing OPL, junctions similar to those described in the younger stages were still present
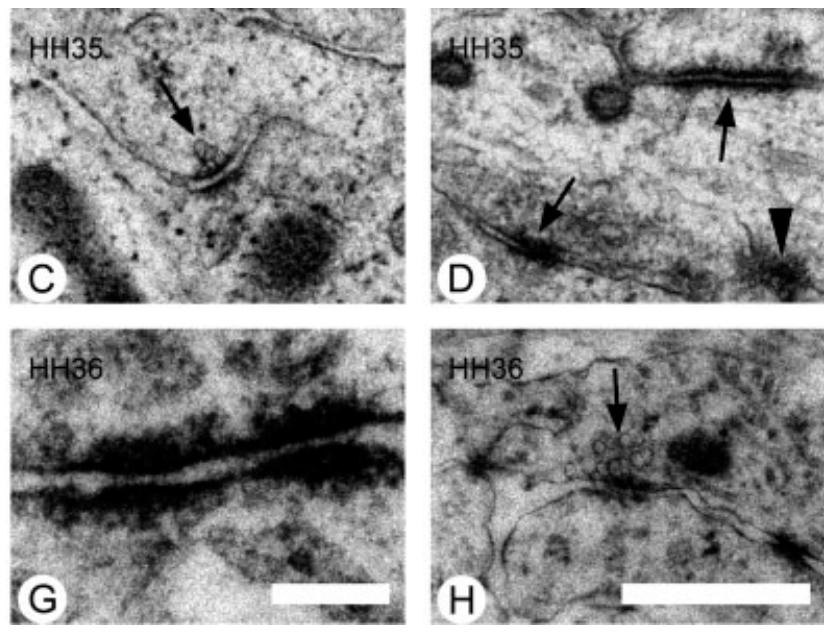

accompanied by synapse-like specializations. In the other contacts, the number of vesicle-like structures was increased (E, arrow). F-H: On E10, the junction (F, G) resembled those observed in the IPL (Figs. 1H and $1 \mathrm{~L}$ ). Lines or bars were visible that crossed the gap between the opposed membranes (F, arrow; enlarged detail in G). The contacts with synapse-like specializations showed an increased number of vesicles $(H$, arrow). Scale bar in $G=0.1 \mu \mathrm{m}$, for all other images in $\mathrm{H}=0.5 \mu \mathrm{m}$.

(Fig. 2F). The gap of such junctions was filled with electron dense material and opaque lines or bars crossed the gap (Figs. 2F and 2G). Some synapse-like contacts in the OPL showed an increased number of vesicle-like structures (Fig. 2H).

\section{HH38 (E12)}

The simple junctions described earlier had become much longer (Fig. 1N) but still smaller junctions also occurred. Such junctions were always devoid of synaptic vesicles. Compared with earlier stages, the junctions appeared to be less frequent at this stage. Contacts showing characteristics related to conventional synapses were again increased in number. The shape of the vesicle-like structures and the appearance of the pre- and postsynaptic densities varied (Figs. 1O-1S). Some contacts displayed spherical or flattened, others pleomorphic vesicles sometimes accompanied by dense core vesicles (Fig. 1Q). In some, pre- or postsynaptic densities were faint or negligible (Fig. 10), others showed thickened pre- or postsynaptic membranes (Figs. 1Q-1S). Also, reciprocal synapses were seen (Fig. 1P). The contacts occurred between cell processes of different structure. Contacts with ribbon-shaped densities were slightly more frequent compared to E10, but their number was still rather low. In some, the length of the ribbons ranged up to $130 \mathrm{~nm}$ (Fig. 1T) and the opposed membranes were mostly darkened.

In the OPL, the situation was as described earlier, except that the frequency of junctions, which showed no vesicle-like structures, was reduced. The frequency of junctions with synapse-like specializations appeared increased. They were relatively uniform and similar to those found at earlier stages. Photoreceptor triad ribbon synapses, characterized by invaginations of postsynaptic profiles, have not been observed. 


\section{DISCUSSION}

The future IPL became visible on E6 and a nascent OPL at the end of E8. First junctions were observed shortly afterwards, in the IPL on E7, in the OPL on E8. The junctions were characterized by closely opposed unit membranes with a small amount of electron dense material at the cytoplasmic side. Frequently, opaque structures or lines were found within or crossing the intercellular gap. These junctions were always devoid of vesicles. Contacts with vesicle-like compartments appeared in both plexiform layers on E8 and increased in number with age. In the IPL, such contacts started to diversify from E9 onward, whereas they remained uniform in the OPL. On E10, contacts with ribbon-like cytoplasmic inclusions associated with vesicle-like structures appeared in the IPL.

\section{Technical Considerations}

The intention of this study was to solve the problem whether or not first synapses form in the retinal plexiform layers when spontaneous activity occurs at the beginning of the second embryonic week. This is primarily a qualitative aspect, which requires a small sample of embryos only. The specimens were treated in the same way and the sections came from a defined region unmistakably identifiable at all ages. Only contacts between neurons and their processes, respectively, were investigated in detail and described here. Muller cells were easily distinguished from neurons by their structure and intense staining. Neuroepithelial cells were not observed in the plexiform layers at the age when first synaptic contacts appeared. The linear extension of the synaptic contacts ranged between about $0.1-1 \mu \mathrm{m}$, only gap junctions measured up to $2.9 \mu \mathrm{m}$ in the older stages. Thus, the prepared serial sections, which covered a distance of at least $1.05 \mu \mathrm{m}$ in the youngest and up to $4.76 \mu \mathrm{m}$ in the older stages, were sufficient for analyses. It should be considered, that the histological procedure and compression during sectioning induced volume changes and influenced also the appearance of synaptic vesicles (Valdivia, 1971).

\section{Early Junctions in the Plexiform Layers}

In earlier studies, junctions in the developing chick plexiform layers were identified as puncta adherentia and maculae adherentes (diminutae), basal junctions, and gap junctions (Fujisawa et al., 1976b; Gleason and Wilson, 1989; Hughes and LaVelle, 1974; Lasansky, 1969; McLaughlin, 1976; Sheffield and Fischman, 1970). In this study, junctions were observed which showed dark stained opposed membranes, separated by a small cleft filled with electron dense material of varying intensity. In most junctions, electron dense material was associated to the membranes at their cytoplasmic side. First junctions were found on E7 (IPL) and on E8 (OPL), shortly after the future plexiform layers have appeared. Their frequency increased with age and many were elongated, the intercellular gap was reduced, and they were always devoid of vesicles. These junctions (IPL: Figs. 1A, 1D, 1H, and 1N; OPL: Figs. 2A, 2B, 2D, and 2F) corresponded to those described earlier as punctate junctions, puncta adherentia, or maculae adherentes (Gleason and Wilson, 1989; Hughes and LaVelle, 1974; McLaughlin,
1976; Sheffield and Fischman, 1970). However, there was no decisive evidence for the presence of the characteristic tonofilaments, distinctive for puncta adherentia or maculae adherentes.

Decisive for identification were opaque lines or bars which crossed the intercellular gap representing tubular-like structures (IPL: Figs. 1H-1J; OPL: Figs. 2F and $2 \mathrm{G}$ ). This resembles the basal junctions depicted by Lasansky (1969) in turtle photoreceptors on the basal surface of the pedicles. Their structure corresponds to gap junctions described by Fujisawa et al. (1976a,b) in the retina of embryonic and newly hatched chicks and by other authors in mammals (Dowling and Boycott, 1966). According to Fujisawa et al. (1976b), gap junctions disappear during the first half of the second embryonic week, whereas specific immunohistochemistry and dye coupling suggested their presence until at least E15 (Becker et al., 2002). The presented morphological evidence confirms that gap junctions are present in both arising plexiform layers. These junctions provide a means to coordinate electrical activity, metabolic, and signaling processes in coupled cells (Kandler and Katz, 1998).

The observed second type of junctions (Fig. 1B), which occurred during the investigated period at about equal frequencies, showed similarities to contacts depicted by Dhingra et al. (2001) in dissociated rat cultures. These contacts maturated to conventional synapses by the addition of synaptic vesicles. Thus, it might be possible that they represent precursors of conventional synapses. However, a definitive conclusion is not possible since a sequential analysis of fixed tissue of subsequent stages is inappropriate to draw conclusions about such a dynamic process.

\section{Chemical Synapses Occur in Both Plexiform Layers on E8}

Conventional chemical synapses are easily identified by their qualifying specializations such as thickened opposing plasma membranes separated by a small cleft filled with electron-dense material and vesicles associated to the presynaptic side of the synapse (Fawcett, 1994). Dyad (and triad) synapses show in addition synaptic ribbons. Contacts with corresponding specializations appeared shortly after the junctions described earlier have emerged.

In the developing IPL, immature conventional synapses were observed from E8 (HH34) onward (Figs. 1C, $1 \mathrm{E}-1 \mathrm{G}, 1 \mathrm{~K}$, and $1 \mathrm{O}-1 \mathrm{~S})$. Comparable synapses were described earlier in the embryonic chick retina or chick retinal explants (Bird, 1986; Gleason and Wilson, 1989; Hering and Kröger, 1996). The frequency of the conventional synapses increased during the investigated period. Already on E9, different types could be distinguished and diversity was particularly noticeable on E12. This diversification may not only indicate the maturation of conventional synapses, but also the increasing complexity of the intraretinal network. On E10 contacts occurred in the IPL with short ribbonshaped densities, already accompanied by vesicles (Figs. 1L, 1M, and 1T). Pre- or postsynaptic densities were not present in the early ribbon synapses, as already observed by Sheffield and Fischman (1970). With age, pre- and postsynaptic densities appeared, the ribbons enlarged, and the number of vesicles increased. 
Dyad synapses were rare on E10 and their frequency increased much less when compared with conventional synapses. The early appearance of chemical synapses stands in contrast to earlier TEM studies, which reported that conventional synapses in the IPL do not form before E12/13 and dyad synapses not before E14/15 (Daniels and Vogel, 1980; Hering and Kröger, 1996; Hughes and LaVelle, 1974; Sheffield and Fischman, 1970).

In the nascent OPL, first immature conventional synapses were found on E8 (HH35), a few hours later than in the IPL. These contacts (Figs. 2C, 2E, and $2 \mathrm{H}$ ) were generally smaller than those observed in the IPL, which refers also to the size of synaptic vesicles. The synapses remained small and relatively uniform until E12. The onset of synaptogenesis in the OPL is described here for the first time. Triad synapses were not observed in this study. It is well documented that photoreceptor cell pedicles start to differentiate on E13 and that triad synapses do not occur before E15 (Hughes and LaVelle, 1974; McLaughlin, 1976).

\section{Backup for an Early Onset of Synaptogenesis}

Different simultaneous events, which are prerequisites for the process of synapse formation or which directly or indirectly indicate the presence of synaptic contacts, support an early onset of synaptogenesis and assume that the discrepancy to earlier TEM studies relates probably to differences in the investigated locations. The lines of evidence are: First, retinal neurons start to leave the cell cycle before the first synapses have been found and generate processes during the time of synapse formation. The first cells that start to leave the cell cycle are ganglion cells (on E2) and bipolar cells (E5) are the last (Prada et al., 1991). Ganglion cells form dendrites from E6 onward; amacrine cells extend processes into the IPL from E7/8, and bipolar cells from E8 onward (D'Alessandri et al., 1995; Karlsson et al., 1998; Prada et al., 1991; Spira et al., 1987). These cell processes start to separate the ganglion cell and inner nuclear layer and thus mark the initiation of the future IPL. From E9 onward amacrine cells begin to form first sublayers in the IPL indicating the presence of different cell types (Drenhaus et al., 2004). The future OPL begins to appear with the extension of processes of horizontal cells from E8 and bipolar cells from E9 onward (D'Alessandri et al., 1995). The process of generation and maturation of retinal cells and the development of the plexiform layers start in the central part of the retina close to the apex of the optic fissure and proceeds symmetrically with respect to the optic fissure into the periphery (Drenhaus et al., 2003; Prada et al., 1991). Because of the asymmetric growth of the retina from HH29 onward, the geometric center in the adult animal locates about $2 \mathrm{~mm}$ further nasally (Rager et al., 1993).

Second, several presynaptic membrane proteins such as the synaptic vesicle protein 2 (SV2), synaptophysin, synaptotagmin, and syntaxin are expressed in the IPL at E7/8 (Bergmann et al., 1999, 2000; Hering and Kröger, 1996). Furthermore, the neuronal calcium sensor-1 (NCS-1), a Ca2+ binding protein, which has been implicated in synaptogenesis and neurotransmission, occurs in the IPL on E7 (Bergmann et al., 2002). In horizontal cell bodies, NCS-1 expression was observed from $\mathrm{E} 7$ onward and from E9 onward in their processes in the OPL (Bergmann et al., 2002). The expression of all of these proteins shows a central to peripheral developmental pattern. Third, neurotransmitters have been involved in the process of retinal development and the formation of intercellular contacts. An array of different endogenously released neurotransmitters (ACh, dopamine/L-Dopa, glycine, GABA, glutamate) were reported to trigger and to modulate calcium waves, which occur in the chick retina from E7/8 on. GABAergic, glutamatergic, muscarinic, nicotinic acetylcholine, and AMPA receptors are present shortly before or during the period when spontaneous activity occurs, spread across the retinal layers, and from central to peripheral retina (Catsicas et al., 1998, 2001; Hering and Kröger, 1996; Kubrusly et al., 2003; Pearson et al., 2002; Prada et al., 1998; Silveira dos Santos Bredariol and Hamassaki-Britto, 2001; Vailati et al., 2003; Yamashita and Fukuda, 1993).

Hence, this study presents evidence that contacts between nerve cells exist when spontaneous activity occurs in the chick retina from E7/8 onward (Catsicas and Mobbs, 2001; Sernagor and Grzywacz, 1996; Wong et al., 1998). They appear shortly after the plexiform layers have started to emerge. Gap junctions, characteristic for the lateral pathway of information processing, are formed before first chemical synapses appear indicating that this pathway starts to establish in advance of the vertical pathway which carries information centrally via chemical synapses from photoreceptors via bipolar cells to ganglion cells (Cook and Becker, 1995; Maslim and Stone, 1986).

\section{ACKNOWLEDGMENTS}

We wish to thank Ms. Monique Carrel for her excellent technical assistance.

\section{REFERENCES}

Becker DL, Bonness V, Catsicas M, Mobbs P. 2002. Changing patterns of ganglion cell coupling and connexin expression during chick retinal development. J Neurobiol 52:280-293.

Bergmann M, Grabs D, Rager G. 1999. Developmental expression of dynamin in the chick retinotectal system. J Histochem Cytochem 47:1297-1306.

Bergmann M, Grabs D, Rager G. 2000. Expression of presynaptic proteins is closely correlated with the chronotopic pattern of axons in the retinotectal system of the chick. J Comp Neurol 418:361-372.

Bergmann M, Grabs D, Roder J, Rager G, Jeromin A. 2002. Differential expression of neuronal calcium sensor-1 in the developing chick retina. J Comp Neurol 449:231-240.

Bird MM. 1986. An ultrastructural study of embryonic chick retinal neurons in culture. Cell Tissue Res 245:563-577.

Catsicas M, Mobbs P. 2001. GABAb receptors regulate chick retinal calcium waves. J Neurosci 21:897-910.

Catsicas M, Bonness V, Becker D, Mobbs P. 1998. Spontaneous Ca2+ transients and their transmission in the developing chick retina. Curr Biol 8:283-286.

Catsicas M, Allcorn S, Mobbs P. 2001. Early activation of $\mathrm{Ca}(2+)$-permeable AMPA receptors reduces neurite outgrowth in embryonic chick retinal neurons. J Neurobiol 49:200-211.

Cook JE, Becker DL. 1995. Gap junctions in the vertebrate retina. Microsc Res Tech 31:408-419.

D’Alessandri L, Ranscht B, Winterhalter KH, Vaughan L. 1995. Contactin/F11 and tenascin-C co-expression in the chick retina correlates with formation of the synaptic plexiform layers. Curr Eye Res 14:911-926.

Daniels MP, Vogel Z. 1980. Localization of $\alpha$-bungarotoxin binding sites in synapses of the developing chick retina. Brain Res 201:4556 . 
Dhingra NK, Reddy R, Govindaiah, Hemavathy U, Raju TR, Ramamohan Y. 2001. Synaptic development in semi-dissociated cultures of rat retina. Int J Dev Neurosci 19:533-540.

Dowling JE, Boycott BB. 1966. Organization of the primate retina: Electron microscopy. Proc R Soc Lond B Biol Sci 166:80-111.

Drenhaus U, Thomas K, Rager G. 2000. The course of later generated axons in the developing optic nerve of the chick embryo. A morphometric electron microscopic study. Brain Res Dev Brain Res 121:35-53.

Drenhaus U, Morino P, Veh RW. 2003. On the development of the stratification of the inner plexiform layer in the chick retina. J Comp Neurol 460:1-12.

Drenhaus U, Morino P, Rager G. 2004. Expression of axonin-1 in developing amacrine cells in the chick retina. J Comp Neurol 468:496-508.

Fawcett DW. 1994. Bloom and Fawcett. A textbook of histology. New York: Chapman \& Hall. $964 \mathrm{p}$

Feller MB, Wellis DP, Stellwagen D, Werblin FS, Shatz CJ. 1996 Requirement for cholinergic synaptic transmission in the propagation of spontaneous retinal waves. Science 272:1182-1187.

Fujisawa H, Morioka H, Nakamura H, Watanabe K. 1976a. Gap junctions in the differentiated neural retinae of newly hatched chickens. J Cell Sci 22:597-606.

Fujisawa H, Morioka H, Watanabe K, Nakamura H. 1976b. A decay of gap junctions in association with cell differentiation of neural retina in chick embryonic development. J Cell Sci 22:585-596.

Gleason E, Wilson M. 1989. Development of synapses between chick retinal neurons in dispersed culture. J Comp Neurol 287:213-224.

Hamburger V, Hamilton HL. 1951. A series of normal stages in the development of the chick embryo. J Morphol 88:49-92.

Hering H, Kröger S. 1996. Formation of synaptic specializations in the inner plexiform layer of the developing chick retina. J Comp Neurol 375:393-405.

Hughes WF, LaVelle A. 1974. On the synaptogenic sequence in the chick retina. Anat Rec 179:297-301.

Kandler K, Katz LC. 1998. Coordination of neuronal activity in developing visual cortex by gap junction-mediated biochemical communication. J Neurosci 18:1419-1427.

Karlsson M, Clary DO, Lefcort FB, Reichardt LF, Karten HJ, Hallbook F. 1998. Nerve growth factor receptor TrkA is expressed by horizontal and amacrine cells during chicken retinal development. J Comp Neurol 400:408-416.

Kubrusly RC, Guimaraes MZ, Vieira AP, Hokoc JN, Casarini DE, de Mello MC, De Mello FG. 2003. L-DOPA supply to the neuro retina activates dopaminergic communication at the early stages of embryonic development. J Neurochem 86:45-54.

Lasansky A. 1969. Basal junctions at synaptic endings of turtle visual cells. J Cell Biol 40:577-581.

Maslim J, Stone J. 1986. Synaptogenesis in the retina of the cat Brain Res 373:35-48.
McLaughlin BJ. 1976. A fine structural and E-PTA study of photoreceptor synaptogenesis in the chick retina. J Comp Neurol 170:347364.

Pearson R, Catsicas M, Becker D, Mobbs P. 2002. Purinergic and muscarinic modulation of the cell cycle and calcium signaling in the chick retinal ventricular zone. J Neurosci 22:7569-7579.

Prada C, Puga J, Perez-Mendez L, Lopez R, Ramirez G. 1991. Spatial and temporal patterns of neurogenesis in the chick retina. Eur $\mathrm{J}$ Neurosci 3:559-569.

Prada F, Medina JI, Lopez-Gallardo M, Lopez R, Quesada A, Spira A, Prada C. 1999. Spatiotemporal gradients of differentiation of chick retina types I and II cholinergic cells: Identification of a common postmitotic cell population. J Comp Neurol 410:457-466.

Prada FA, Quesada A, Dorado ME, Chmielewski C, Prada C. 1998 Glutamine synthetase (GS) activity and spatial and temporal patterns of GS expression in the developing chick retina: Relationship with synaptogenesis in the outer plexiform layer. Glia 22:221-236.

Rager U, Rager G, Frei B. 1993. Central retinal area is not the site where ganglion cells are generated first. J Comp Neurol 334:529-544.

Sernagor E, Grzywacz NM. 1996. Influence of spontaneous activity and visual experience on developing retinal receptive fields. Curr Biol 6:1503-1508.

Sernagor E, Eglen SJ, O’Donovan MJ. 2000. Differential effects of acetylcholine and glutamate blockade on the spatiotemporal dynamics of retinal waves. J Neurosci 20:RC56:1-6.

Sheffield JB, Fischman DA. 1970. Intercellular junctions in the developing neural retina of the chick embryo. Z Zellforsch Mikrosk Anat 104:405-418.

Silveira dos Santos Bredariol A, Hamassaki-Britto DE. 2001. Ionotropic glutamate receptors during the development of the chick retina. J Comp Neurol 441:58-70.

Spira AW, Millar TJ, Ishimoto I, Epstein ML, Johnson CD, Dahl JL Morgan IG. 1987. Localization of choline acetyltransferase-like immunoreactivity in the embryonic chick retina. J Comp Neurol 260:526-538.

Vailati S, Moretti M, Longhi R, Rovati GE, Clementi F, Gotti C. 2003. Developmental expression of heteromeric nicotinic receptor subtypes in chick retina. Mol Pharmacol 63:1329-1337.

Valdivia O. 1971. Methods of fixation and the morphology of synaptic vesicles. J Comp Neurol 142:257-273.

Vaney DI, Nelson JC, Pow DV. 1998. Neurotransmitter coupling through gap junctions in the retina. J Neurosci 18:10594-10602.

Wong WT, Sanes JR, Wong RO. 1998. Developmentally regulated spontaneous activity in the embryonic chick retina. J Neurosci 18:8839-8852

Yamashita M, Fukuda Y. 1993. Calcium channels and GABA receptors in the early embryonic chick retina. J Neurobiol 24:1600-1614.

Zhou ZJ. 1998. Direct participation of starburst amacrine cells in spontaneous rhythmic activities in the developing mammalian retina. J Neurosci 18:4155-4165. 